infundibulo-pelvic pedicle stump, followed by the development of the retroperitoneal space with the identification of the umbilical artery, the iliac vessels and laterally the psoas muscle with the genito-femoral nerve. Paravesical and pararectal spaces are developed down to the pelvic floor. A radical pelvic lymphadenectomy is performed bilaterally. Once lymph node involvement is excluded, we proceed to parametrectomy.

Radical parametrectomy is started with the dissection from the posterior leaf of the broad ligament. The anterior division of the internal iliac artery (IIA) is identified and the uterine artery and vein are transected proximally at their origin using a vascular sealing system or Hem-O-Lok clips. The ureters are then mobilized from their attachments and separated from the medial leaf of the peritoneum down to the ureteral tunnel below the uterine artery and to their entrance into the bladder. Aided by the vaginal probe, the bladder peritoneum is incised, and the bladder is dissected and mobilized inferiorly down to the middle third of the vagina. After dissection of the bladder pillar, the vesico-vaginal space is joined to the paravesical space, completely separating the bladder from the anterior vaginal wall. In cases of anatomical distortion or bladder adhesions, instillation of $300 \mathrm{cc}$ of saline solution associated with Methylene blue dye in the bladder might be required to guide the dissection. Posteriorly, the peritoneum is incised at the level of the cul-de-sac of Douglas and the rectovaginal space is developed isolating the uterosacral ligaments. The proximal parametrium and para-vaginal tissues are finally dissected as in a Type B1 Querleu Morrow radical hysterectomy.

The same procedure is performed on both sides. A circular incision is made about $3 \mathrm{~cm}$ below the vaginal cuff aided by upward vaginal traction.

Results When compared to Radiation therapy RP presents a lower rate of late complications, making it the preferred approach to treat younger patients. Traditionally performed via laparotomy, minimally invasive approach is now proven feasible and effective.

Conclusion This article presents a focused anatomic review and describes the surgical technique of the five-port robotic assisted radical parametrectomy.

Disclosures Eric Lambaudie and Gilles Houvenaeghle report grants and personal fees from Intuitive Surgical, outside the submitted work.

The other authors have no other conflicts of interest to declare.

\section{ENHANCED RECOVERY AFTER SURGERY (ERAS) FOR PARA-AORTIC LYMPHADENECTOMY-A NEW TREND TO CONSIDER?}

${ }^{1}$ Isabelle Masquin, ${ }^{2}$ Houssein EL Hajj, ${ }^{1}$ Christophe Zemour, ${ }^{1}$ Camille Jauffret Farra, ${ }^{1}$ Guillaume Blache, ${ }^{1}$ Mellie Heinemann, 'Laura Sabiani, ${ }^{1}$ Clement Brun, ${ }^{1}$ Gilles Houvenaeghel, ${ }^{1}$ Marion Faucher, ${ }^{1}$ Eric Lambaudie. ${ }^{1}$ Paoli Calmettes Institute; ${ }^{2}$ Centre Léon Bérard

\subsection{6/ijgc-2020-ESGO.46}

Background Enhanced recovery after surgery (ERAS) is a multimodal approach aiming to improve rehabilitation after surgery. In gynecologic malignancies, para-aortic lymphadenectomy (PAL) is indicated for either diagnostic or therapeutic finalities. Minimally invasive surgery (MIS) constitutes the cornerstone for ERAS programs.

Methods This retrospective study conducted between November 2006 and January 2018, aims to analyze the role of ERAS implementation for patients undergoing PAL. Starting 2016, an ERAS protocol was implemented for all the patients in our institution. All patients who underwent PAL for gynecologic malignancies were included in this study. To analyze the impact of this implementation on the surgical outcomes (length of hospital stay (LOS)) and the post-operative complications, we compared the patients who underwent PAL within ERAS protocol between 2016 and 2018 'ERAS Group' to the patients who underwent PAL prior to this implementation (between 2006 and 2015) 'Prior to ERAS group'.

Results A total of 193 patients were identified. 'ERAS Group' was associated with a significant decrease of median LOS (2 days vs. 3 days, $\mathrm{p}<0.001)$ and a significant increase in earlier post-operative discharges: $\mathrm{OR}=29.62$ [13.58-64.64], $\mathrm{p}<0.001$. Two factors were independently associated with early postoperative discharge: Implementation of the ERAS protocol $(\mathrm{OR}=25.64$ [8.14-80.71], $\mathrm{p}<0.0001)$ and the endorsement of the extraperitoneal technique for $\mathrm{PAL}(\mathrm{OR}=5.92 \quad[2.10$ 16.68], $\mathrm{p}=0.0008)$. There was no difference in intra-operative complications rate between groups $(\mathrm{p}=0.497)$. More postoperative complications were found in the 'ERAS group' $(23 \%$ vs $10 \%, \mathrm{p}=0.017$ ) but this difference was not significant for severe complications $(\mathrm{p}=0.277)$ and lymphocele rate $(\mathrm{p}=0.248)$.

Conclusions Implementing ERAS protocols for patients undergoing minimally invasive PAL is an independent factor improving early recovery and decreasing the LOS without increasing severe complications.

Disclosures Drs Isabelle Masquin, Mellie Heinemann, Christophe Zemmour, Camille Jauffret-Fara, Guillaume Blache, Laura Sabiani, Clément Brun, Marion Faucher and Houssein El Hajj have no conflicts of interest or financial ties to disclose. Gilles Houvenaeghel and Eric Lambaudie are proctors for Intuitive Surgical.

\section{Diagnostics}

\section{A NEW PROPOSAL FOR THE CLINICAL CLASSIFICATION OF VULVAR LICHEN SCLEROSUS: AN OBSERVATIONAL PROSPECTIVE STUDY}

${ }^{1}$ Veronica Boero, ${ }^{2}$ Carlo A Liverani, ${ }^{3}$ Massimiliano Brambilla, ${ }^{1}$ Ermelinda Monti ${ }^{4}$ Filippo Murina, ${ }^{5}$ Roberto Tozzi, ${ }^{6}$ Eugenia DI Loreto, ${ }^{1}$ Chiara Maria F Dell'utri, ${ }^{1}$ Giada Libutti, ${ }^{7}$ Fabio Parazzini. ${ }^{1}$ Fondazione Irccs Ca' Granda - Ospedale Maggiore Policlinico; Gynecologic Unit; ${ }^{2}$ Humanitas San Pio X; Department of Gynecology; ${ }^{3}$ Fondazione Irccs Ca' Granda - Ospedale Maggiore Policlinico; Plastic Surgery Service, Gynecology Unit; ${ }^{4}$ Ospedale Vittore Buzzi; Lower Genital Tract Disease Unit, Obstetrics and Gynecology Department; ${ }^{5}$ Women's Centre (Level 3), John Raddliffe Hospital; University of Oxford; Department of Gynaecologic Oncology, Nuffield Department of Women's and Reproductive Health (Wrh); ${ }^{6}$ Fondazione Irccs Ca' Granda - Ospedale Maggiore Policlinico; Department of Gynecology; ${ }^{7}$ University Hospital of Milan; Department of Clinical Sciences and Community Health

\subsection{6/ijgc-2020-ESG0.47}

Introduction/Background Vulvar Lichen Sclerosus (VLS) is a chronic inflammatory disorder which commonly affects the female anogenital epithelium, leading to scarring, anatomical 
distortions, impaired sexual function, decreased quality of life and increased vulvar cancer risk.

An agreement to measure VLS severity in a standard way is yet to be defined and, to our knowledge, no standardized clinical classification of anatomical modifications in VLS has been validated.

The purpose of this study was to prepare a clinical classification for VLS aimed at defining the morphological patterns of this condition, while stratifying them into grades. The classification is intended to provide a homogeneous and reproducible description of the different features of this disease. It

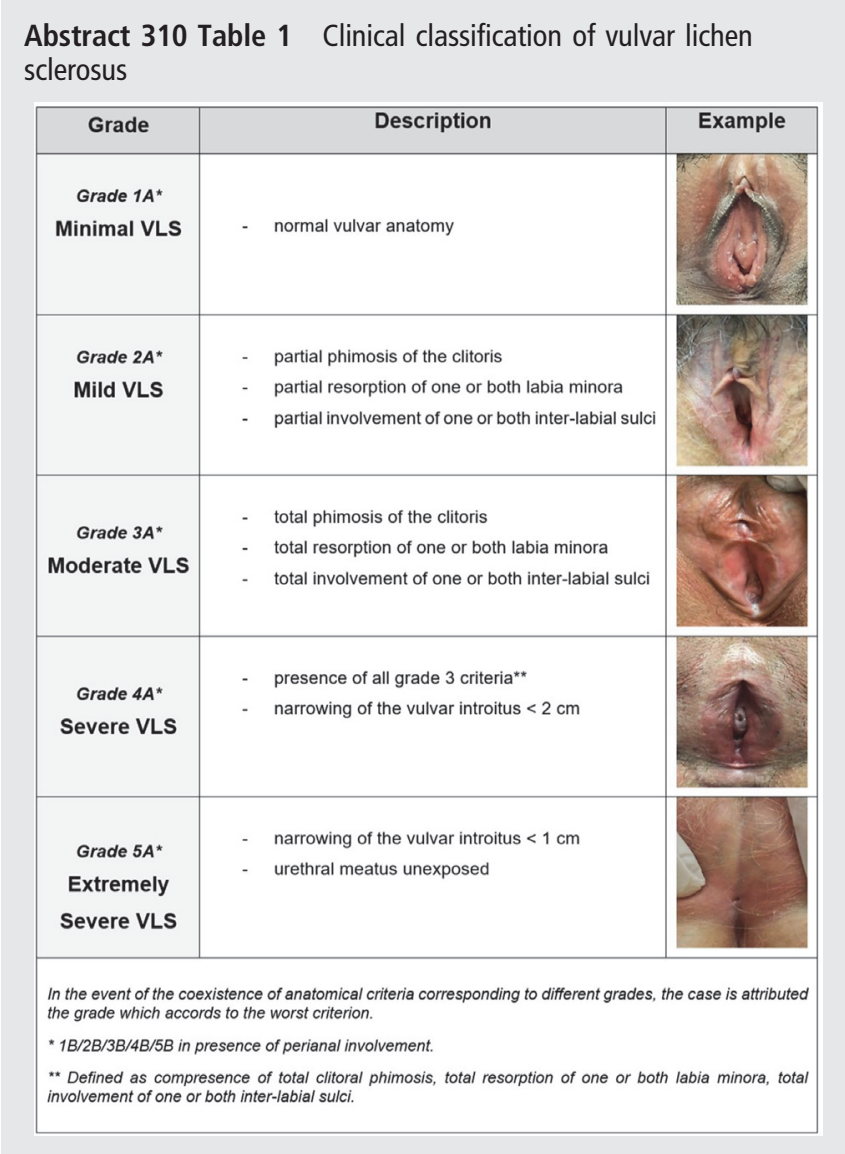

Abstract 310 Table 2 Level of agreement among the raters

\begin{tabular}{|c|c|c|c|c|}
\hline \multicolumn{5}{|c|}{ Agreement among the raters for the 137 cases of VLS } \\
\hline \multicolumn{4}{|c|}{ ICC } & $95 \% \mathrm{Cl}$ \\
\hline \multicolumn{2}{|l|}{ Total panel } & \multicolumn{2}{|c|}{0.89} & $0.87-0.91$ \\
\hline \multicolumn{2}{|c|}{ Board members } & \multicolumn{2}{|c|}{0.92} & $0.89-0.93$ \\
\hline \multicolumn{2}{|c|}{ Gynecologists with no expertise } & \multicolumn{2}{|c|}{0.87} & $0.84-0.90$ \\
\hline \multicolumn{2}{|c|}{ Cases in women aged $<50$ years } & \multicolumn{2}{|c|}{0.92} & $0.86-0.96$ \\
\hline \multicolumn{2}{|c|}{ Cases in women aged $>50$ year } & \multicolumn{2}{|c|}{0.89} & $0.86-0.92$ \\
\hline \multicolumn{5}{|c|}{ Agreement among the raters between the first and the second evaluation. } \\
\hline & Board member 1 & Board member 2 & $\begin{array}{l}\text { Gynecologist no } \\
\text { expertise } 1\end{array}$ & $\begin{array}{c}\text { Gynecologist no } \\
\text { expertise } 2\end{array}$ \\
\hline $\begin{array}{l}\text { Kappa } \\
\text { (95\% Cl) }\end{array}$ & $0.91(0.79-1.00)$ & $0.96(0.87-1.00)$ & $0.79(0.60-0.95)$ & $0.91(0.79-1.00)$ \\
\hline $\begin{array}{l}\text { ICC } \\
(95 \% \mathrm{CI})\end{array}$ & $0.97(0.94-0.99)$ & $0.99(0.97-0.99)$ & $0.93(0.86-0.97)$ & $0.97(0.94-0.99)$ \\
\hline
\end{tabular}

also serves as an important tool for the evaluation of the course of the disease over time, response to treatment, and for comparison of clinical studies.

Methodology A board of seven specialists with expertise in vulvar pathology were asked to outline the anatomical criteria for the definition of VLS severity (phimosis of the clitoris, resorption of the labia minora, involvement of the inter-labial sulcus, and narrowing of the vulvar introitus), identifying five grades to be used to build-up of a score model. The classification was validated by 13 physicians upon pictures of 137 consecutive patients. Each physician individually assigned a grade to each case, according to the abovementioned criteria. Interrater agreement among evaluators was analysed by means of ICC (Intraclass Correlation Coefficient). Intra-observer reproducibility and inter-observer concordance in vivo were analysed by means of Kappa index.

Results This study provides a new classification of VLS, based on defined anatomical criteria and graded into mutually exclusive progressive classes (table 1).

The ICC analysis showed a substantial agreement in the attribution of the grade of VLS among the 137 cases, ICC $=0,89$ (0.87-0.91), both in the expert and in the nonexpert group (ICC $=0.92$ and 0.87 respectively). An 'almost perfect' agreement was achieved for intra-observer reproducibility and among physicians in vivo (Kappa 0.93).

Conclusion Our classification showed a high accuracy in defining morphological modifications in VLS. It is easy to use, reproducible, and can be applied by different health care providers in daily clinical practice and in all clinical settings.

Disclosure Nothing to disclose.

\section{DIAGNOSTIC VALUE OF HE4, CA-125, ROMA AND CPH-I FOR PREOPERATIVE ASSESSMENT OF OVARIAN TUMORS}

${ }^{1}$ Núria Carreras Diéguez, ${ }^{1}$ Ariel Glickman, ${ }^{1}$ Núria Agustí, ${ }^{2}$ Marta Del Pino, ${ }^{1}$ Berta DiazFeijoo, 'Jaume Pahisa, 'Pere Fusté, 'Aureli Torne. ${ }^{1}$ Hospital Clínic de Barcelona; Gynecologic Oncology Unit; ${ }^{2}$ Hospital Clínic; Hospital Clinic Barcelona; Gynaecology

\subsection{6/jgc-2020-ESGO.48}

Introduction/Background Among patients with adnexal masses, preoperative identification of epithelial ovarian cancer (EOC) or metastatic cancer in the ovary (MCO) is essential for surgical planning. Our aim was to assess the performance of CA125, HE4, and the probability models, Risk of Ovarian Malignancy Algorithm (ROMA) and Copenhagen Index (CPHI), to preoperatively identify EOC or MCO.

Methodology We performed a single center retrospective study including women who underwent surgery for an ovarian tumor between January 2000 and December 2018. We defined two study groups: one group comprising women with benign pathology and borderline epithelial ovarian tumors and a second group comprising women with EOC or MCO. We computed sensitivity, specificity and predictive values of CA125, HE4, ROMA and CPH-I at different cutoff points. We performed receiver operative curve analysis for tumor markers, CPH-I and ROMA models. We performed subgroup analysis including only premenopausal, postmenopausal women, stage I EOC and women harboring ovarian tumors with inconclusive diagnosis of malignancy by ultrasound features.

Results One thousand seventy-one patients were included, $852(79.55 \%)$ presented benign or borderline epithelial 